ORIGINAL ARTICLE

\title{
Nosocomial cutaneous abscesses in septic infants
}

\section{Mandel, Y Littner, F B Mimouni, S Dollberg}

See end of article for

Arch Dis Child Fetal Neonatal Ed 2004;89:F161-F162. doi: 10.1136/adc.2002.011668

authors' affiliations

.....................

Correspondence to:

Dr Dollberg, Department

of Neonatology, Lis

Maternity Hospital, Tel

Aviv-Sourasky Medical

Center, 6 Weizman Street,

Tel Aviv, 64239, Israel;

dollberg@tasmc.health. gov.il

28 November 2002

Objective: To retrospectively study the epidemiology of nosocomial cutaneous abscesses in 46 consecutive septic infants.

Results: Ten infants had one abscess or more. Surviving infants with abscesses had a longer duration of bacteraemia, which disappeared within 24 hours of drainage.

Conclusion: Infants with persistent bacteraemia should be examined regularly for the presence of abscesses.
$\mathrm{P}$ ersistent bacteraemia in infants may be due to bacterial endocarditis, osteomyelitis, focal infection, inadequate treatment with antibiotics, ${ }^{1}$ and an infected central venous catheter. ${ }^{2}$ We have observed that nosocomial cutaneous abscesses in infants are associated with persistent bacteraemia. A Medline search using the terms "abscess" and "neonate" or "newborn infant" failed to reveal any article. We report the epidemiology of nosocomial sepsis with cutaneous abscesses.

\section{PATIENTS AND METHODS}

From 1 July 1997 to 31 December 1999, of 1185 admissions to our neonatal intensive care unit, all 46 consecutive infants with nosocomial - that is, acquired in hospital after birthneonatal sepsis were studied. The diagnosis required both an unwell infant and one positive blood culture. Some 29\% had persistent bacteraemia. We excluded all early ( 3 days or less after birth) onset sepsis and late sepsis perinatally transmitted, such as late onset group B streptococcal disease. Cutaneous abscesses were defined as subcutaneous collections with inflammation (swelling, tenderness, redness, and localised heat) that produced pus on drainage. Persistent bacteraemia was defined as positive blood cultures obtained on receipt of the results of the first blood culture, growing the same bacteria with similar antibiogram.

A retrospective chart review collected the variables depicted in tables 1 and 2. For analysis, preterm and prolonged rupture of membranes were defined as $<38$ weeks gestation and $>18$ hours respectively.

\section{RESULTS}

Forty six of the 1185 infants admitted (3.9\%) had nosocomial sepsis. Ten $(22 \%)$ had cutaneous abscesses, diagnosed on day 12-32 of life. In all, bacteraemia preceded the abscess diagnosis. Obstetrical characteristics of infants with and without abscesses were similar, in terms of premature rupture of the membranes and mode of delivery (data not shown). Neonatal clinical and laboratory characteristics are depicted in tables 1 and 2. Thirteen (28\%) infants died. One had a cutaneous abscess. All infants who had cutaneous abscesses were clinically unwell, and seven of them had persistent bacteraemia. The blood cultures remained positive until the diagnosis of the abscess in five of the infants. Infants who died and infants who survived were analysed separately because it is meaningless to study bacteraemia duration in infants who died.

Eight infants had only one abscess. One infant had three simultaneous abscesses and one had two. No abscesses were diagnosed in non-bacteraemic infants. In seven of the 10 infants with abscesses, bacteraemia was persistent. Abscesses were mostly on the limbs $(n=11)$, one on the forehead, and one on the umbilical stalk. Although infants had a complete physical examination daily, there was a gap of up to 24 days between onset of sepsis and abscess recognition. Infants with abscesses had higher gestational age, but did not differ significantly from those without abscesses in terms of the other variables considered. No infant received a central venous catheter, except for the initial placement of the umbilical venous catheter, which was removed in all cases before the septic episode. Pathogens recovered from the pus matched that of the blood in only four of the abscesses. They were mostly Gram negative rods (two were Escherichia coli, four were Klebsiella pneumonia, two were Serratia marcescens, one was Enterobacter cloacae, and one Pseudomonas

Table 1 Clinical characteristics

\begin{tabular}{lll}
\hline & $\begin{array}{l}\text { With abscess } \\
(\mathbf{n}=10)\end{array}$ & $\begin{array}{l}\text { Without } \\
\text { abscess }(\mathbf{n}=36)\end{array}$ \\
\hline Birth weight (g) & $1490(782)$ & $1134(614)$ \\
Gestational age (weeks) & $31(4)^{*}$ & $26(6)$ \\
1 min Apgar & $7(0-9)$ & $5(1-9)$ \\
5 min Apgar & $9(4-10)$ & $8(5-10)$ \\
Age at sepsis (weeks) & $10(4)$ & $12(10)$ \\
Presence of UVC & $3(30 \%)$ & $28(78 \%)$ \\
Duration of UVC (days) & $0(0-3)^{* *}$ & $4(0-8)$ \\
Presence of UAC & $7(70 \%)$ & $30(83 \%)$ \\
Duration of UAC (days) & $3(2.5)$ & $4(2.3)$ \\
Mechanical ventilation & $7(70 \%)$ & $34(90 \%)$ \\
Days of ventilation & $4(3)$ & $10(10)$ \\
TPN & $9(90 \%)$ & $36(100 \%)$ \\
Duration of TPN (days) & $8(2.3)$ & $9(8)$ \\
Received blood transfusions & $6(60 \%)$ & $28(78 \%)$ \\
Presence of PDA & $7(70 \%)$ & $25(96 \%)$ \\
Appropriate therapy according & $10(100 \%)$ & $35(97 \%)$ \\
to antibiogram & & \\
\hline
\end{tabular}

Values are presented as mean (SD) or $n(\%)$, except for Apgar scores and duration of UVC and UAC which are expressed as median (range). ${ }^{*} \mathrm{p}<0.017 ;{ }^{* *} \mathrm{p}<0.004$ compared with infants without abscesses. UVC, Umbilical venous catheter; UAC, umbilical arterial catheter; TPN, total parenteral nutrition; PDA, patent ductus arteriosus. 
Table 2 Pertinent laboratory characteristics

\begin{tabular}{lll}
\hline & $\begin{array}{l}\text { With abscess } \\
(\mathbf{n}=10)\end{array}$ & $\begin{array}{l}\text { Without abscess } \\
(\mathbf{n}=36)\end{array}$ \\
\hline White blood cells $\left(\times 10^{3} / \mu \mathrm{l}\right)$ & $34.8(18.3)$ & $27.4(22.2)$ \\
Absolute granulocytes $\left(\times 10^{3} / \mu \mathrm{l}\right)$ & $14.8(17.6)$ & $16.7(17.5)$ \\
Platelets $\left(\times 10^{3} / \mu \mathrm{l}\right)$ & $95(124)^{*}$ & $247(159)$ \\
$\mathrm{pH}$ at onset of sepsis & $7.31(0.04)$ & $7.26(0.11)$ \\
Pco 2 at onset of sepsis $(\mathrm{mm} \mathrm{Hg})$ & $43(8)$ & $47(12)$ \\
Duration of bacteraemia (days) & $6(6-20)^{* *}$ & $4(1-9)$ \\
\hline
\end{tabular}

Data are expressed as mean (SD), except the duration of bacteraemia which is median (range).

${ }^{*} p<0.012 ;{ }^{* *} p<0.03$. Kruskal Wallis and $t$ tests were used to study differences between infants with and without abscesses. Backward stepwise regression analysis was used to study the independent impact of these variables on mortality. $p<0.05$ was considered significant.

aeruginosa) and one Gram positive coccus (Streptococcus gamma hemolyticus).

Surviving infants with cutaneous abscesses had longer duration of bacteraemia and lower platelet counts at the onset of infection than infants without abscesses. They had significantly shorter duration of the umbilical venous catheter $(\mathrm{p}<0.004)$. Blood cultures became negative within 24 hours of abscess drainage in all infants. Echocardiograms, performed in three infants with abscesses, and bone scans, performed in two, were negative.

In backward stepwise regression analysis, infants with abscesses had bacteraemia for longer and lower platelet counts independent of birth weight (or gestational age) and adequacy of antibiotic treatment.

\section{DISCUSSION}

Persistent bacteraemia appropriately treated according to the antibiogram must prompt the search for occult foci or an infected central venous catheter. We showed that cutaneous abscesses were found in $22 \%$ of infants after an episode of nosocomial infection.

The absence of abscesses in infants who died early could be because they had overwhelming systemic (rather than focal) infection. Alternatively, early death prevented late abscess development. Infants with abscesses had higher gestational age, which may have made them more likely to survive a protracted bacteraemic course and may explain the shorter duration of the umbilical venous catheter.

Bacteria obtained from pus culture were not always the same as those found at blood culture. The abscess and the bacteraemia may have been unrelated, which is not likely as blood cultures became sterile within 24 hours of drainage. Alternatively, pus in antibiotic treated patients is often sterile, and a positive culture (at times with mixed pathogens) may represent skin contamination.

The mechanism of thrombocytopenia in sepsis is not well understood; it is believed that platelet consumption in the microcirculation and platelet associated IgGs are important contributing factors. ${ }^{3-5}$ Nevertheless, abscesses were strongly associated with lower platelet counts, the reason for which remains to be established.

Abscesses may have formed at the port of entry of the infection ${ }^{6}$ or metastatic foci. The first scenario may have been present in eight infants, as the abscess sites were compatible with intravenous infusion sites (despite aseptic insertion techniques). The delay between sepsis and abscess occurrence renders the causal relation difficult to establish. All abscesses were discovered, in spite of a meticulous search, up to 24 days after sepsis onset; this and the presence of multiple simultaneous abscesses in two infants support the scenario of haematological seeding.

Persistent bacteraemia in infants should prompt vigilance and regular, active search for cutaneous abscesses, in addition to echocardiography to exclude bacterial endocarditis. Drainage of the abscess may be rapidly effective in suppressing the source of bacterial seeding.

\section{Authors' affiliations}

D Mandel, Y Littner, F B Mimouni, S Dollberg, Department of Neonatology, Lis Maternity Hospital, Tel Aviv Sourasky Medical Center, Tel Aviv, Israel, and the Sackler Faculty of Medicine, Tel Aviv University, Tel Aviv, Israel

\section{REFERENCES}

1 Noel GJ, Edelson PJ. Persistent bacteremia [letter]. Pediatrics 1990;86:145-6.

2 Craft A, Finer N. Nosocomial coagulase negative staphylococcal (CoNS) catheter-related sepsis in preterm infants: definition, diagnosis, prophylaxis, and prevention. J Perinatol 2001;21:186-92.

3 Stephan F, Cheffi MA, Kaplan C, et al. Autoantibodies against platelet glycoproteins in critically ill patients with thrombocytopenia. Am J Med 2000; 108:554-60.

4 Patic $\mathrm{CH}$, Lazarchick J. The effect of bacteremia on automated platelet measurements in neonates. Am J Clin Pathol 1990;93:391-4.

5 Gill FM. Thrombocytopenia in the newborn. Semin Perinatol 1983;7:201-12.

6 Stamm WE, Kolff CA, Dones EM, et al. A nursery outbreak caused by Serratia marcescens: scalp-vein needles as a portal of entry. J Pediatr 1976;89:96-9 